\title{
A TECHNIQUE FOR ADVANCING ENERGY EFFICIENCY IN 5G MASSIVE MIMO FOR MOBILE WIRELESS NETWORK
}

\author{
Aaishwarya Soni ${ }^{1}$, Mrs. Megha Soni ${ }^{2}$ \\ Student of M.Tech (Digital Communication), Electronics and communication engineering, Babulal Tarabai Institute of \\ Research and Technology, Sagar (M.P.), India ${ }^{1}$
}

HOD and Guide, Department of Electronics and communication engineering, Babulal Tarabai Institute of Research and Technology, Sagar (M.P.), India ${ }^{2}$

\begin{abstract}
The immensely improving the advancement of upcoming generations, the fifth-generation wireless technology continuously developed and extent to more progressive. The field of mobile technology offers improved network capacity, small latency, excessive availability, additional reliability with dynamic speed. This paper presents an outline of fifth-generation wireless technology. In recent times, energy cost and its involvement to global carbon dioxide emissions are evolving as key concerns, which is a severe problem for cellular networks. Moreover, Energy Efficiency (EE) is an enormous approach that massively declines the circuit power consumption in fifth-generation cellular networks. This significance is enhanced due to the expanding number of antennas in massive multiple-input multiple-output (MIMO) systems. Energy efficiency has now become a major aspect in context of communication networks. With the advent of the fifth generation of wireless networks, with millions of additional base stations and billions of connected devices, the demand for energy-efficient system design and operation will be even more fascinating. Further, this paper emphases in simulating an area enclosed by random implementation of small cells to serve hundreds of users i.e., Massive MIMO in the simulation area. The outcomes illustrates that the energy efficiency exploiting operation point might be very spectrally inefficient, and the efficient energy can be significantly enhanced by increasing the base station density. Thus, Massive MIMO is a promising solution for maximal energy efficiency implementation.
\end{abstract}

Keywords: Fifth generation, Massive MIMO, small cells, base station, Energy Efficiency (EE).

\section{I.INTRODUCTION}

Gradually, there is an expansion in the mobile networks introducing from the first generation in 1980. We are forthwith awaiting the advent of the 5th generation mobile networks. The first generation $(1 \mathrm{G})$ mobile networks were introduced in 1980, which provided speeds of up to $2.4 \mathrm{kbps}$. It uses analog signals and offers analog voice services. The $1 \mathrm{G}$ network is set up on analog signals/protocol technology (FM) that figures out one predominant issue such as susceptibility to interference, which declines call quality. Additionally, there was an inadequacy of security due to no advanced encryption methods encompassed in analog signals. The $1 \mathrm{G}$ is entangled by limited capacity, huge phone size, lack of voice quality, less battery life, less secure and frequent call dropping. It provides up to $30 \mathrm{KHz}$ spectrum flexibility.

After the first generation, the second generation (2G) mobile network developed on GSM standards was introduced. The second generation used digital signals. Their data speeds were up to $64 \mathrm{kbps}$. It provides various features such as text messages, picture messages, and multimedia messages. Requirements of strong digital signals were a drawback for the second-generation mobile networks. In addition, they were unable to handle complex data such as videos. Between second generation and the third generation, the $2.5 \mathrm{G}$ was introduced. It provides features such as web browsing and email services. It provides speeds of the range 64 to $144 \mathrm{kbps}$.

The Third generation (3G) mobile networks introduced data transmission speeds of $2 \mathrm{Mbps}$. It support broadband and multimedia services. It employs a new technology called UMTS. Urged slightly by the growth in internet and IP network technology, 3G architecture strengthens support for an embellished data rate (throughput speed) and QoS. 3G technology involves the services such as global roaming and enhanced voice quality. It enables mobile telephony. Due to the consumption of more power as compared to previous generations, it is less economical to set up and operate.

Thus, it is a major drawback in 3G Technology. It provides features such as high-speed web browsing, video conferencing, 3D gaming, and mobile TV. Evolution of generations of the communication standards demonstrated in Fig. 1. 


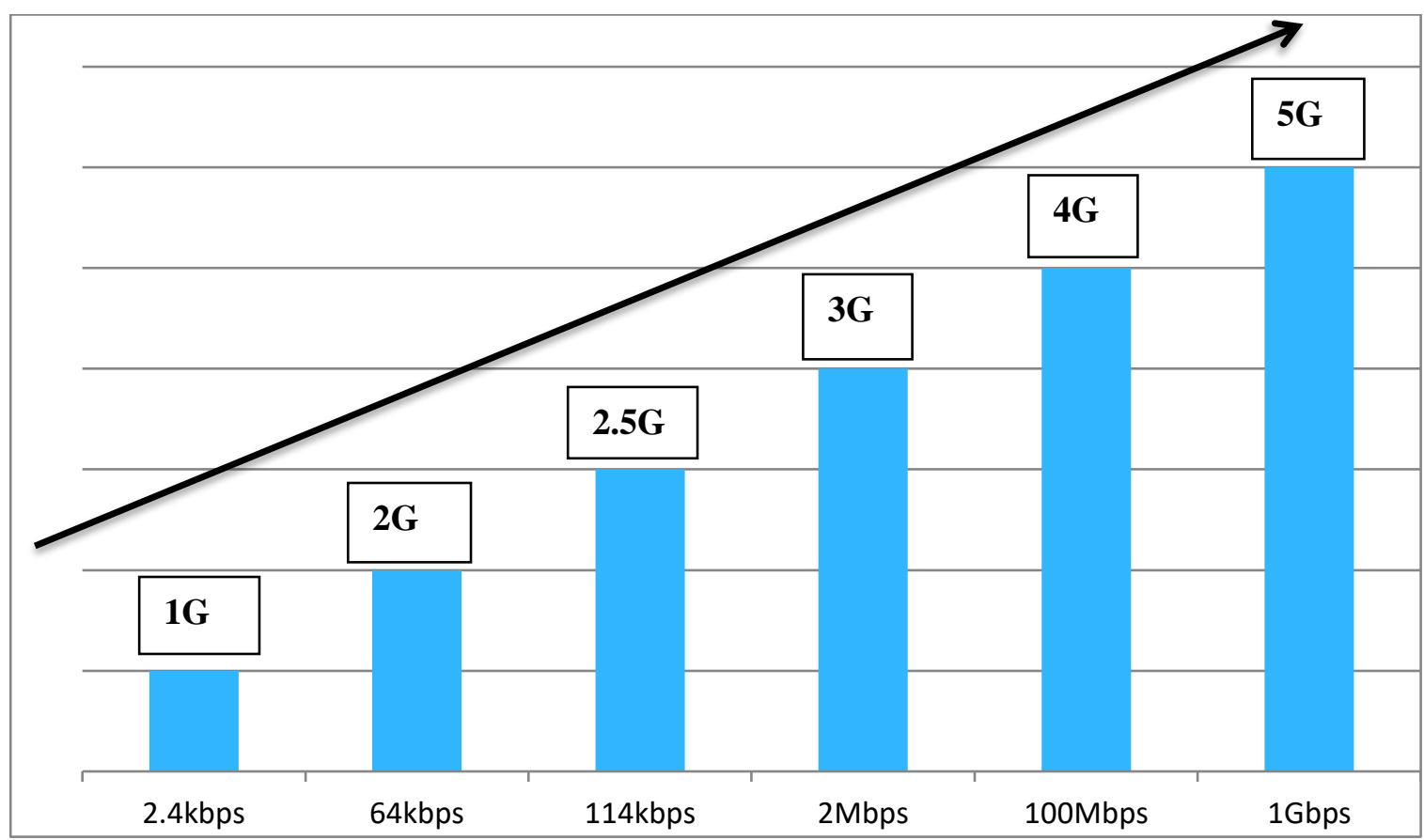

Fig.1 Evolution of generations of the communication standards.

Furthermore, 3G UMTS is backward adaptable with prior generations of cellular wireless technologies through its capacity to endure in distinction with the estate GSM or AMPS technology. The progression from UMTS through High-Speed Packet Access (HSPA) and expanded HSPA (HSPA+) significantly brought enlarged end-to-end network performance and ultimately brought to the development of the next generation of networks i.e. $4 \mathrm{G}$.

The Fourth generations (4G) mobile networks are capable of providing data speeds of up 200 Mbps.It provide broadband data transmission and broadcasting. They offer high security, high quality of service (QoS) and minimal latency. The areas of multicasting and interference mitigation are other major enhancements brought by the 4G era. It involves multicasting services i.e. evolved Multimedia Broadcast Multicast Service (eMBMS). The 4G technology provides the fastest speed, reduced latency, and crystal-clear voice calls.

With the initiation of the state of the art, in the Fifth generation $(5 \mathrm{G})$ mobile network, the world is going to authenticate an ultra-high speed data rate of 1 Gbps. From $1 \mathrm{G}$ to $5 \mathrm{G}$, a thriving increment in the data rates can be observed. Day by day, there is an expansion in the number of mobile users. In addition to this, it desires higher data rates to meet the requirements for online gaming, video streaming, and mobile computing. These applications are highly sensitive to deal with. To comply with these needs in the current state-of the art technology, either the radio power can be increased or the number of remote radio nodes can be increased. Any of these methods will broaden the power requirements.

Using more power implies an increase in the carbon footprint. In such a way, an increment in power contributes to the greenhouse effect. Due to the enormous developments in the field of wireless communication, there is a huge growth in the demand for energy efficiency. The appropriate energy efficiency spectrum is minimal. This factor leads to spectrum inadequacy. Corresponding to the FCC, even under these circumstances, the maximal spectrum efficiency in the current state-of the art technology is only between 15 to $35 \%$. Therefore, the spectrum needs to be consuming efficiently.

Cell sizes will have a major impact on the power requirements in a wireless network. A study shows that the power requirement can be greatly reduced by choosing a smaller cell size. Furthermore, studies also shows that the Radio Access Nodes (RANs) are the most power-consuming component in a wireless network. Therefore, we need better data rates and low power and with the minimum spectrum.

Every ten years or so, new generation of mobile technology comes along. Each generation has set out to fix the flaws of its predecessor. GSM fixes security weaknesses of analogue telephoning. 3G was meant to sort out GSM's lack of 
mobile data and given it did in month succeed $4 \mathrm{G}$ was needed to finally make consuming data less of an unpleasant experience.

\section{II.REQUIREMENT OF ENERGY EFFICIENCY}

\section{A. Why we need Energy Efficiency?}

Recently, energy cost and its contribution to global carbon dioxide emissions are emerging as major concerns, which is a severe problem for cellular networks. Networks need to be energy focused on two fronts:

- $\quad$ It reduced energy consumption of all network resources in order to reduce the operational costs.

- $\quad$ Further, it increased the battery life to enable massive deployment of sensor networks.

Energy efficiency is the cost of energy consumed to achieve spectral efficiency. Furthermore, spectral efficiency will remain a major challenge for the success in early roll out of 5G. In general, there is a enough spectrum and $5 \mathrm{G}$ is alone about optimizing the use of spectrum. However, spectral efficiency doesn't necessarily imply energy efficiency. They can be a tradeoff, just like a tradeoff between energy efficiency and time energy efficiency and cost. Thus, we need an improved energy efficiency as well as spectral efficiency, without compromising the user's quality of service.

B. Why does $5 \mathrm{G}$ system require energy efficiency?

The 5G system require to being energy efficient in order to provide massive connections from various terminals like Internet of Things with diverse quality of requirements. 5G systems must have tremendous spectral and energy efficiency improvements simultaneously. While increasing the network capacity, 1000 fold can be achieved by a large number of small cells, large-scale antennas and much wider bandwidth in the millimeter wave bands. Reducing energy consumption at the same time is still very challenging.

Since, $\mathrm{CO} 2$ emission and operational costs are important. Also, energy efficient transmission originates from energy constrained networks such as wireless sensor networks, ad hoc networks and satellite communications, where wireless devices are powered by batteries that and non-rechargeable or hard to rechargeable.

- $\quad$ EE for Mobile Devices: With the recent explosively growing demand on mobile multimedia communications, the battery energy constraint has become a major concern for smart phones. This motivates optimization towards improving the energy efficiency of mobile devices.

- $\quad$ EE for Communication network: To reduce $\mathrm{CO} 2$ emission and support sustainable development, the resource allocation towards improving the energy efficiency of networks has drawn considerable attention.

\section{Device to Device communication}

With the expanding trend of proximity based applications such as peer to peer pressuring, local multi casting, and advertising. Device to device communications has been recommended to advance local flexibility, network throughput and reinforce public safety services, in case of lack of network coverage 3GPP LTE Advanced. It carries out proximity gain, reuse gain, and hop gain, which enhances network spectral efficiency and device energy efficiency. Through proper mode selection, energy efficient devices and networks can be significantly improved. A radio technology that assists the progress of devices to transmit data specifically with other devices, without transmitting the data using a network framework refers to Device-To-Device (D2D) communication. It is the crucial solution for the next generation (5G) networks to confer with these considerations. D2D communication in cellular networks supports forthright communication between two mobile users without the engagement of a Base Station (BS) or infrastructure-based network. It significantly plays a crucial role in forthcoming cellular networks as it provides ultra-low latency for communication among users. This innovative proposal may operate on a licensed or unlicensed spectrum. It is an innovative development of the conventional cellular communication criterion.

\section{III.ENERGY EFFICIENCY IN NEW TECHNIQUES AND DEPLOYMENT}

A. Network design and implementation: 'Energy efficiency' (EE) is an immense approach that decreases the circuit power consumption (CPC) in fifth-generation cellular networks. This issue was raising because of the enhancing number of antennas in massive multiple-input multiple-output (MIMO) systems. The energy efficiency of wireless networks is advanced by the approach of network designing and implementation. It is an approach employed to implement infrastructure nodes to extent the confined area per consumed energy, rather than just the confined area. In addition, the use of base station (BS) switch-on switch-off algorithms and antenna muting techniques to accommodate the traffic conditions can significantly lower energy consumption. Diverse technologies have been aimed for the planning, implementation and operation of $5 \mathrm{G}$ networks to negotiate with the extensive number of connected devices.

- $\quad$ Extensive Heterogeneous Networks: The idea of extensive networks is a concern with the extremely rising number of devices to assist by expanding the amount of implement infrastructure hardware. The principle fundamentals of network densification can attain strength and emerges as a very strong phenomenon for the implementation of 5G networks. Far from existing network implementation, which consistently decomposes a macro-cell into almost less 
number of smaller areas, each compensates with small base stations, extensive heterogeneous networks significantly enhance the number of infrastructure nodes per unit of area [1]. A huge number of heterogeneous infrastructure nodes differ from macro BSs to femto-cells and relays. Further, it opportunistically implemented and stimulated in a demandbased pattern. Thus, it leads to an irregularly shaped network design such as that shown in Fig. 2.

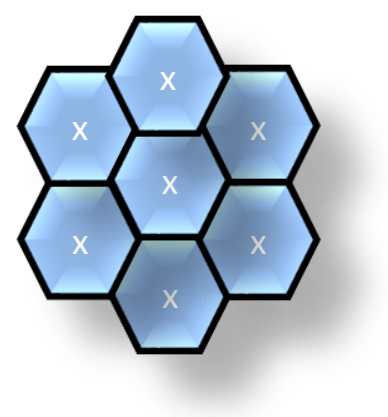

(a)

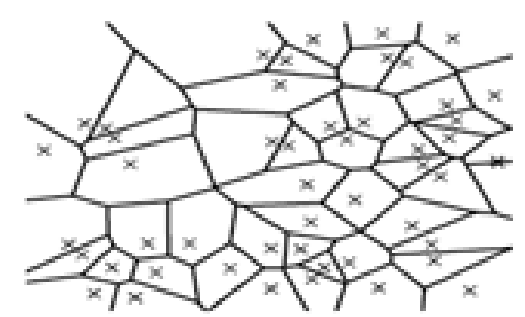

(b)

(a.) Conventional layout

(b.) $5 \mathrm{G}$ layout.

Fig. 2 Evolution of cellular network layout.

A vital dispute when dealing with extensive heterogeneous networks is the design of the arrangement of the nodes in the network, which is usually typical to accomplish inevitably. How cell splitting reduce power requirement? To answer this question, we should first analysis the cell architecture. In case of large cells, high power radio nodes were placed at the cell center. Direct transmission of signals to long distances can cause serious transmission losses because of signal attenuation and fading effects. In addition, there is a large possibility of losing line of sight with the mobile unit. To overcome this losing, large signal power is required. There are serious limitations for this solution. Alternatively, cell splitting can become better in this scenario. Now the question is up to how much level we can do cell splitting. The cells are very small and moving mobile unit can get affected.

The unit often needs to switch to new nodes. This introduces an overhead of multiple handshakes. The combination of different cell sizes can sell better in this scenario. Considering all these aspects, researchers are now focusing on a network that can use different power levels for transmission. Heterogeneous Networks (HetNets) make use of multiple types of access nodes in a wireless network. HetNets offer low power requirement and reduced greenhouse effect. Heterogeneous networks offer high spectrum efficiency, better energy efficiency, and quality of service (QoS). In addition to the conventional high power antenna, HetNet introduce low power antennas. The high power antenna can serve a larger geographical area and the low power antenna can serve a comparatively smaller geographical area.

The traditional networks use high powers for covering larger distances. This can cause serious transmit power losses. In heterogeneous networks, the low power networks serving smaller geographical area can serve mobile units in its vicinity. A number of these low power networks are used to effectively in order to reduce transmission losses, achieve better data rates, and thus, the connectivity can be established in a better way. The high power networks can serve mobile units in its close vicinity. In contrast with connecting to distant HPN, connecting to a nearby LPN can reduce power requirement for the mobile unit and thus, offers a prolonged battery life.

In traditional mobile networks, each antenna is connected with the central gateway. But in heterogeneous networks, there is no need to connect each node with the gateway. Instead, the low power nodes can connect with a high power node and the high power node can connect to the central gateway. The mobile unit in the low power network can get signal from HPN and LPN, if the mobile units rely on only downlink power for connecting to the radio node, always connected with a high power node. Alternatively, if the mobile unit awaits only on the uplink power associated with the radio node, if they get connected with a low power node. But strong interference may appear during downlink from high power node. To escape these situations, mobile units in heterogeneous networks should acknowledge both uplink and downlink power. The signal from large power nodes can broaden into the low power network. This can lead to interference.

To overcome this problem, researchers introduced an idea of using multiple frequencies in the same radio node. The frequency bands have subdivided into two parts F1 and F2. If parameter $\beta$ is used for this division, the first band if one 
Vol. 10, Issue 7, July 2021

DOI 10.17148/IJARCCE.2021.10786

uses $\beta$ times $F$ and the second band F2 uses 1- $\beta$ times $F$. Keeping $\beta<1$, the previous single frequency band can now serve as two different frequency bands. One band is transmitted at a low power compared to the other effective $\alpha$ represents the reduction factor. For better optimization of the technique, $\alpha$ and $\beta$ are to be chosen properly. The traditional network nodes are statically located with the baseband.

In Heterogeneous networks, coordination is maintained between different nodes participating in data transmission. With such cooperation, the pool of baseband can be dynamically shared and can make a better utilization of the resource. Each layer may need to give up some resources in a coordinated fashion to improve performance. Analysts are now dealing with the likelihood of using $\mathrm{L}$ different layers which works may assign us one day to accomplish speeds of the order of terabytes per second. Heterogeneous networks integrate nodes of several power levels. The power requirements can be brought down and this improves energy efficiency. In the heterogeneous network of massive MIMO, improving EE is a big challenge. An area can be under the coverage of a massive MIMO, and from the EE aspect, researchers are still challenging the massive MIMO and HetNets deployment for similar coverage. More flexible and greater EE can be brought by sleep mode control for various small cells in HetNets on the other hand the massive MIMO cell can execute less number of small cells because of its large array gain. Consequently, to study energy-efficient massive MIMO systems in the Heterogeneous networks are required.

\section{B. Energy efficiency in Massive MIMO}

MIMO was another technology of attention for potential candidate for millimetre wave band in 5G, which is likely to arrive in a big way with fighting. MIMO stands for Multiple Input Multiple Output envisages a scenario where antennas are set up at both transmitter and receivers end both set of equipments have 10s or even 100 more antennas that translates into better data rates for users and helps with both spectral and energy efficiency for operators. But there is a challenge designing a scheme that is energy efficient. The following issues needs to be analyzed in future:

- $\quad$ Power Consumption Model and Hardware Impairment:

$>\quad$ Frequency band decide the power model of RF front-end devices. Hence, it is hard to measure and model the energy efficiency for Massive MIMO system.

$>\quad$ Impact of the hardware impairment on energy efficiency in MIMO systems hasn't been evaluated yet.

- $\quad$ EE of Massive MIMO systems in HetNets:

$>\quad$ Given an area, a Massive MIMO cell or a set of small cells from the perspective of energy efficiency can cover it.

$>\quad$ Deployment of Massive MIMO and heterogeneous networks for the same coverage is still being debated.

$>\quad$ Sleep mode control in heterogeneous can provide more flexibility and higher energy efficiency for large number of small cells. While, a Massive MIMO cell performs better than a small number due to its array game and, these energy efficient massive MIMO systems in heterogeneous networks are worth further study.

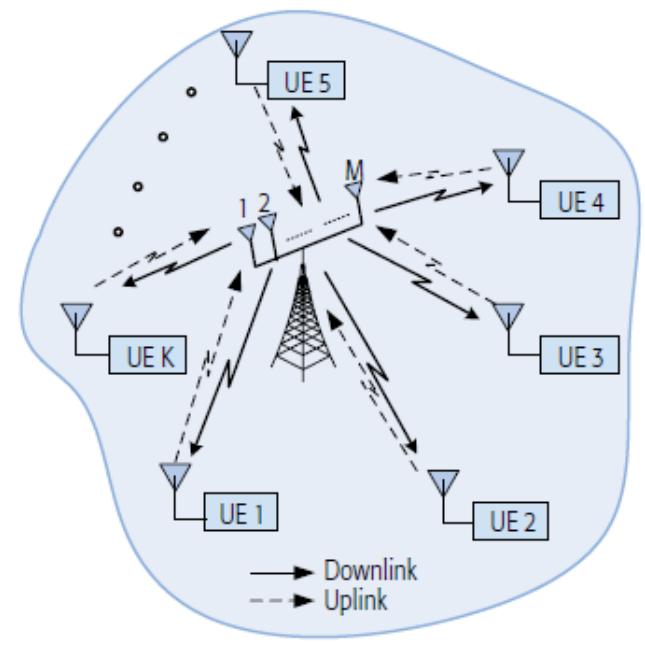

Fig. 3 Massive MIMO communication

\section{A. LITERATURE REVIEW}

\section{IV.RELATED WORKS}

The use of heterogeneous networks a new approach for a substantial extension of the network capacity, with enough cost to provide a rising user experience, where small base stations (BSs) are covered within the core network depend on coverage demand. Energy Efficiency is a crucial parameter that provides a vital design criterion, as it permits 
DOI 10.17148/IJARCCE.2021.10786

operations to endure practically affordable through controlled energy consumption levels. The orthogonal pilot sequences for all UEs in the same cell prevent channel estimation from affected by the interference of other UEs. Additionally, to achieve an appropriate performance system with a massive data rate were investigated, such that it modifies the data rate to diminish inter-cell interference and deliver equivalent channel estimation [2]. The summary of various previous related data, its features, and limitations is shown in Table I.

\section{TABLE I SUMMARY OF PREVIOUS RELATED DATA, ITS FEATURES AND LIMITATIONS}

\begin{tabular}{|c|c|c|c|}
\hline Year & Problem related to & Features & Limitations \\
\hline $\begin{array}{l}2011 \\
{[3]}\end{array}$ & $\begin{array}{l}\text { Problem of pilot } \\
\text { contamination in } \\
\text { Multi-cell systems. }\end{array}$ & $\begin{array}{l}\text { - Used training pilot } \\
\text { sequences. } \\
\text { - Achievable data rate by } \\
\text { developing a multi-cell MMSE. }\end{array}$ & ZF and MMSE. \\
\hline $\begin{array}{l}2013 \\
{[4]}\end{array}$ & $\begin{array}{l}\text { Pilot contamination } \\
\text { effect, the equivalent } \\
\text { System model. }\end{array}$ & $\begin{array}{l}\text { - Used equivalent channel } \\
\text { with pilot. } \\
\text { - } \quad \text { Derived the rate in the } \\
\text { lower bound. }\end{array}$ & Infinite NoA. \\
\hline $\begin{array}{l}2014 \\
{[5]}\end{array}$ & $\begin{array}{l}\text { EE aspects and the } \\
\text { associated gains of } \\
\text { implementing RAN-as-a- } \\
\text { Service }\end{array}$ & $\begin{array}{l}\text { - low complexity decoding } \\
\text { framework } \\
\bullet \quad \text { analysis of sparse code } \\
\text { multiple access (SCMA) scheme. } \\
\text { - } \quad \text { evolution of the LTE-A } \\
\text { network toward 5G infrastructure }\end{array}$ & $\begin{array}{l}\text { - Seamless coverage } \\
\text { and successful delivery of } \\
\text { the control signaling to all } \\
\text { UE in C-RAN is limited } \\
\text { - Lagrange Dual } \\
\text { Decomposition method is } \\
\text { proposed. }\end{array}$ \\
\hline $\begin{array}{l}2015 \\
{[6]}\end{array}$ & $\begin{array}{l}\text { - Pilot assignment } \\
\text { problem. } \\
\text { - Number of activated } \\
\text { antennas and power } \\
\text { allocation units. }\end{array}$ & $\begin{array}{l}\text { - Used joint pilot } \\
\text { assignment and resource allocation. } \\
\text { - Used an iterative } \\
\text { algorithm to solve the transformed } \\
\text { problem. }\end{array}$ & $\begin{array}{l}\text { - Proposed } \\
\text { Algorithm } \\
\text { scheme. }\end{array}$ \\
\hline $\begin{array}{l}2015 \\
{[7]}\end{array}$ & $\begin{array}{l}\text { To maximize the EE the } \\
\text { receiver. }\end{array}$ & $\begin{array}{l}\text { Used ADC resolutions of } \\
\text { a multi-antenna receiver }\end{array}$ & $\begin{array}{l}\text { - Impact of quality } \\
\text { of channel estimation } \\
\text { should be carefully } \\
\text { investigate. }\end{array}$ \\
\hline $\begin{array}{l}2016 \\
{[8]}\end{array}$ & $\begin{array}{l}\text { Evaluate the performance } \\
\text { of user mobility for } 5 \mathrm{G} \\
\text { small cell networks }\end{array}$ & $\begin{array}{ll}\text { - } & \text { Using individual mobility } \\
\text { model } & \text { (IMM). }\end{array}$ & $\begin{array}{l}\text { System model } \\
\text { based on IIM. }\end{array}$ \\
\hline $\begin{array}{l}2016 \\
{[9]}\end{array}$ & $\begin{array}{l}\text { Pilot contamination. } \\
\text { Increased CPC. }\end{array}$ & $\begin{array}{l}\text { Maximized EE under } \\
\text { optimised NoA, number of users, } \\
\text { and pilot sequences. }\end{array}$ & $\begin{array}{l}\bullet \quad \text { Proposed (AO-BS) } \\
\text { algorithm }\end{array}$ \\
\hline $\begin{array}{l}2017 \\
{[10]}\end{array}$ & $\begin{array}{l}\text { To achieve multiple } \\
\text { spectral and EE gains. }\end{array}$ & $\begin{array}{l}\text { B } \quad \text { By designing the BSs with } \\
\text { several antennas. }\end{array}$ & $\begin{array}{l}\text { RF energy } \\
\text { harvesting capabilities } \\
\text { cannot investigated. }\end{array}$ \\
\hline $\begin{array}{l}2019 \\
{[11]}\end{array}$ & $\begin{array}{l}\text { Inter-cell interference. } \\
\text { High computational } \\
\text { complexity. }\end{array}$ & $\begin{array}{l}\text { - Maximized EE under } \\
\text { optimal NoA selection, and user } \\
\text { scheduling. }\end{array}$ & $\begin{array}{ll} & \text { Adaptive Markov } \\
\text { Chain. } & \\
\text { - } & \text { Limited policies } \\
\text { generalized to MM regime. }\end{array}$ \\
\hline
\end{tabular}

\section{V.SYSTEM MODELE AND METHODOLOGY}

This paper analyzes by virtue of how to enhance network efficiency with uniform energy efficiency (EE), which this design examines in two approaches. The early one is to increase the system capacity when employing distinctive cells size with the identical EE of the system. Another one is to enhance the capacity of the system with the same EE when user density of the system advances and how to enhance the number of small cells in the network, in order to have the capacity involved without further installation of base stations (BS), which means additional BS in the system. Thus, more routing features, more Intra-tier intervention in association with distinct small cells and more energy used in order to enhance capacity.[12][13]

This paper intensified on three objective prospective approaches:

- $\quad$ to examine energy efficiency conceptions, features, and design restraints of UDNs 
Vol. 10, Issue 7, July 2021

DOI 10.17148/IJARCCE.2021.10786

- $\quad$ to investigate hardware impairments and their effect on massive MIMO

- $\quad$ to evaluate and examine the network capacity and energy efficiency for UDNs and how the network density alters the EE.

A. Random waypoint mobility model

1) Mobile Ad hoc Networks (MANET) are infrastructure-less wireless networks and are predominantly deployed in necessity situations such as arenas and railways. Unlike antenna-based or wired networks, the use devices like mobile phone for a direct communication without any intermediate in a deployed area .MANET is a compelling network in which each device functions as a node and accompanies a certain to random mobility pattern.[14]

2) Mobility demonstrates the arrangement of each node with esteem to the position of other nodes at distant with respect to time. The nodal connectedness revolves around the corresponding position of each node with respect to the position of other nodes. Sequentially, it affects the data rate, signal strength, and the shortest nodal path. Mobility pattern is accordingly a compelling factor that can affect network performance. Some of the designed popular mobility patterns are Gauss-Markov Mobility Model, Random Waypoint Mobility Model, Random Walk, and City Selection Model [15].

3) The most prevalent is the Random Waypoint Mobility Model in which each node is meant to choose an arbitrary terminal. The node moves towards the selected location in a consecutive line at a randomly selected homogeneous speed. Upon attaining the target, the node ceases for a stated duration known as pause time [15] and then endures to recite its movement. Each node is executed within an established area, where it selects its destination and move randomly within the preferred area. The typical connectivity is achieved after each lantern interval. MANETS are broadly simulated employing RWP models since they resemble the position where people can use mobile phones within a given area and correspondingly move randomly within the same area. With the randomized movement of each node, connectivity develops into an issue that must be solved. Consequently, in this consideration, an RWP model was used. RWP models does not give a homogeneous distribution node in the network, instead, the nodes are clustered midway in the region they move[16] and technique of investigating and simulating the use of ultra-dense networks to assess the energy efficiency of 5G UDNs has been emphasized.

The simplest methodology is to rely on heavy Monte Carlo simulations. Efforts in this direction can be found in [17] and [18].Acknowledging the arbitrary implementation of base stations, the coverage probabilities of UDNs are determined for small-cell users. The simulation was executed under different values of SNR in order to examine the consequence of the SNR value on the EE when a small cells composition is used. Moreover, the EE is examined under different values of path loss coefficient $(\alpha)$ and different number of massive MIMO antennas. The simulation is performed by MATLAB.

\section{B. Operation Metrics}

The operation metric for this paper is the energy efficiency (EE) examined as an applicable and relevant metric, which plays a very extensive role in determining the gains attained by carrying out energy efficient approaches in wireless cellular networks. It is used to assess the energy consumption per information bit that is well transmitted over the network. It is measured in joules per bit.

$$
\text { Energy Efficiency }=\frac{\text { Energy for successfully transmitted bits }}{\text { total energy }}
$$

The conventional simulation specifications are constructed as $\mathrm{S}=3 \times 3 \mathrm{~km} 2$. The area of the simulation with user density $\lambda \mathrm{u}$ alters from 1 to 105 per square kilometer. Also, transmit power of BS corresponds to Ps $=0.05 \mathrm{~W}$ with path loss exponent $\alpha=[3,4]$ for comparatively lossy environments and for the state of full specular reflection from the earth surface, assumption of small scale fading $\mu=1$, variance of Gaussian noise $\sigma 2=0.001$ and the bandwidth of single channel $\mathrm{B}=100 \mathrm{KHz}$. The BS density differs from 1 to 104 per square kilometer and the level of hardware impairments is between 0 to 0.2 . 
The flowchart of the following simulation process is given in Fig. 4.

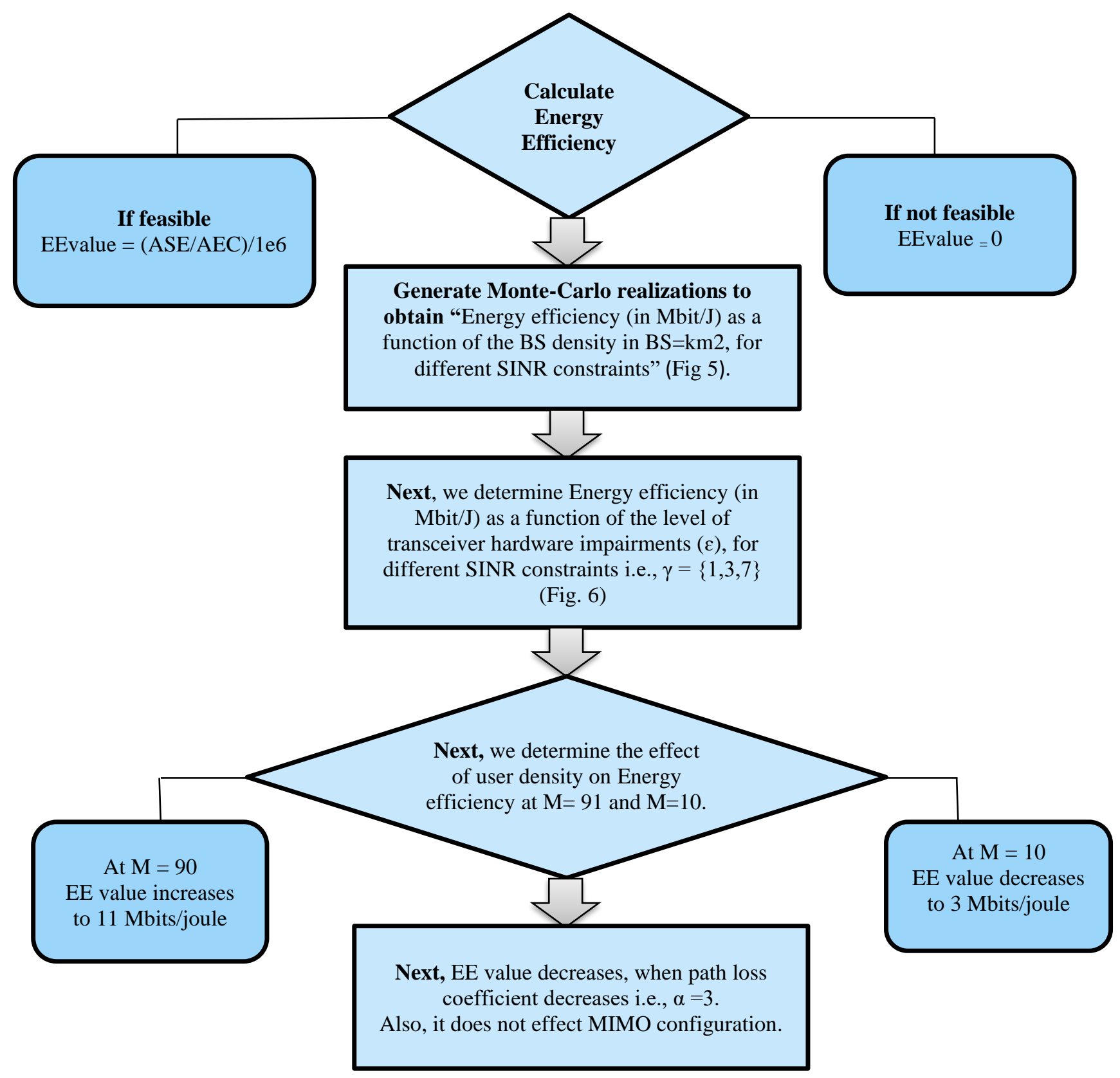

Fig. 4 Flowchart of the simulation process.

In state of massive MIMO simulation, number of antennas is 10 and 91 . The simulation is executed for three values of SNR equals $[1,3,7]$. The hardware simulation parameters are shown in Table II. Whereas, table III contain system simulation parameters. The simulation is performed by MATLAB software. 
International Journal of Advanced Research in Computer and Communication Engineering

Vol. 10, Issue 7, July 2021

DOI 10.17148/IJARCCE.2021.10786

TABLE II. HARDWARE SIMULATION PARAMETERS AND VALUES

\begin{tabular}{|c|c|c|}
\hline Hardware parameters & Notations & Values \\
\hline Coding/decoding/backhaul & A & $1.15^{*} 10^{\wedge}-9[\mathrm{~J} / \mathrm{bit}]$ \\
\hline Static power consumption & C0 & $10 \mathrm{~W}^{*} \tau[\mathrm{J} / \mathrm{symbol}]$ \\
\hline Circuit power per active user & $\mathrm{C} 1$ & $0.1 \mathrm{~W}^{*} \tau[\mathrm{J} / \mathrm{symbol}]$ \\
\hline Circuit power per BS antenna & D0 & $0.2 \mathrm{~W}^{*} \tau[\mathrm{J} / \mathrm{symbol}]$ \\
\hline Signal processing coefficient & D1 & $1.56^{*} 10^{\wedge}-9[\mathrm{~J} / \mathrm{symbol}]$ \\
\hline
\end{tabular}

TABLE III. SYSTEM SIMULATION PARAMETERS AND VALUES

\begin{tabular}{|c|c|c|}
\hline System Parameter & Notation & Values \\
\hline User density & $\mu$ & $\begin{array}{c}\text { 1-105 per square } \\
\text { kilometer }\end{array}$ \\
\hline $\begin{array}{c}\text { Transmit power } \\
\text { of BS }\end{array}$ & Ps & $0.05 \mathrm{~W}$ \\
\hline $\begin{array}{l}\text { Path loss } \\
\text { exponent }\end{array}$ & $\alpha$ & 3,4 \\
\hline Fading & $\mu$ & 1 \\
\hline $\begin{array}{c}\text { Variance of } \\
\text { Gaussian noise }\end{array}$ & $\sigma 2$ & 0.001 \\
\hline $\begin{array}{l}\text { Bandwidth of } \\
\text { single channel }\end{array}$ & B & $100 \mathrm{KHz}$ \\
\hline $\begin{array}{c}\text { Base station } \\
\text { density }\end{array}$ & $\lambda$ & $\begin{array}{l}\text { 1-104 per square } \\
\text { kilometer }\end{array}$ \\
\hline $\begin{array}{l}\text { Level of hardware } \\
\text { impairments }\end{array}$ & $\epsilon$ & $0-0.2$ \\
\hline SINR & $\gamma$ & $1,3,7$ \\
\hline $\begin{array}{l}\text { Number of } \\
\text { antennas }\end{array}$ & M & 10,91 \\
\hline Number of user per cell & $\mathrm{K}$ & 100 \\
\hline Coherence block length & $\mathrm{S}$ & 400 \\
\hline Propagation loss at $1 \mathrm{~km}$ & $\omega$ & $130 \mathrm{~dB}$ \\
\hline Power amplifier efficiency & $\eta$ & 0.39 \\
\hline Symbol time & $\tau$ & $1 / 2^{*} 10^{\wedge} 7[\mathrm{~s} / \mathrm{symbol}]$ \\
\hline
\end{tabular}

\section{VI.SIMULATION RESULT}

A. Optimizing the Energy Efficiency

Three different SINR constraints are considered in Fig. 5, $\gamma \in\{1,3,7\}$ which corresponds to the average SEs log2 $(1+\gamma)$ $\epsilon\{1,2,3\}$. In all three cases, the EE is computed using both the lower bound on the average SE in Proposition -: If MRC is employed, a lower bound on the UL average SE [bit/symbol/user] is

$$
\frac{M\left(1-\epsilon^{2}\right)^{2}}{\left(K+\frac{\sigma^{2}}{\rho}\right)\left(1+\frac{2}{\beta(\alpha-2)}+\frac{\sigma^{2}}{\rho}\right)+\frac{2 K}{\alpha-2}\left(1+\frac{\sigma^{2}}{\rho}\right)+\frac{K}{\beta}\left(\frac{4}{(\alpha-2)^{2}}+\frac{1}{\alpha-1}\right)+M\left(1-\epsilon^{2}\right)\left(\frac{1}{\beta(\alpha-1)}+\epsilon^{2}\right)}
$$

and an upper bound obtained by averaging over the instantaneous SE derived using Monte Carlo simulations (This is an upper bound since we only consider an average of 1000 closest interfering BSs, while the exact result requires an infinite number of interferers in R2). 
Vol. 10, Issue 7, July 2021

DOI 10.17148/IJARCCE.2021.10786

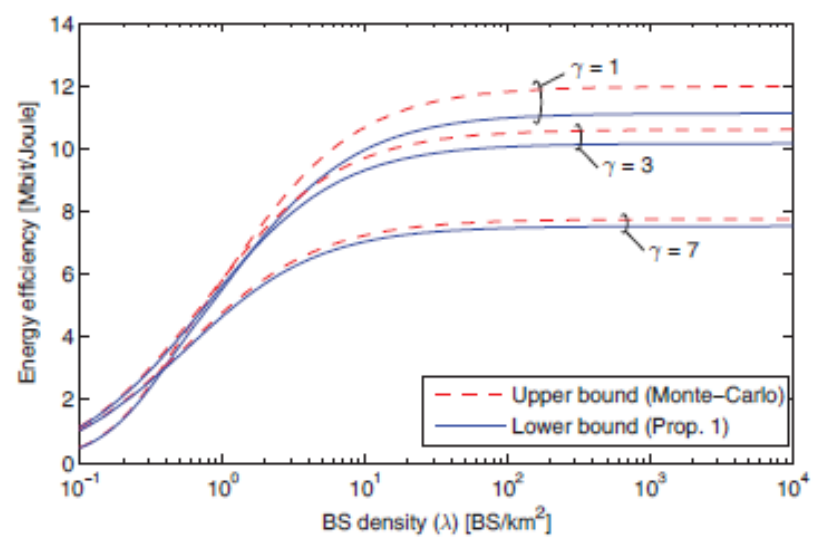

Fig.5 Energy efficiency (in Mbit/J) as a function of the BS density in BS=km2, for different SINR constraints .

Several important observations can be made from the results presented in Fig. 5. Firstly, there is only a small gap (that reduces as takes larger values) between the lower and upper bounds $\omega$, and the curves behave exactly the same for any value Secondly, the EE can be greatly improved by increasing the BS density, meaning that small cells are a promising solution for maximal EE deployment.

B. Significance of Transceiver hardware Impairments:

Eventually, we examine the saturation reign where $\lambda \longrightarrow \infty$ and demonstrate the influence of transceiver hardware impairments on the EE. Fig. 6 displays the EE as a function of $\varepsilon$, the standard of hardware impairments. The loss is minimal for $\gamma=1$, but it can be approximately large when increases. The outcomes of Fig. 6illustrate that for the examined network the EE loss due to hardware impairments is insignificant for $\varepsilon \leq 0.1$ when $\gamma \longrightarrow\{1,3\}$. We may accomplish that moderate levels of hardware impairments have a slight impact on networks configuration.

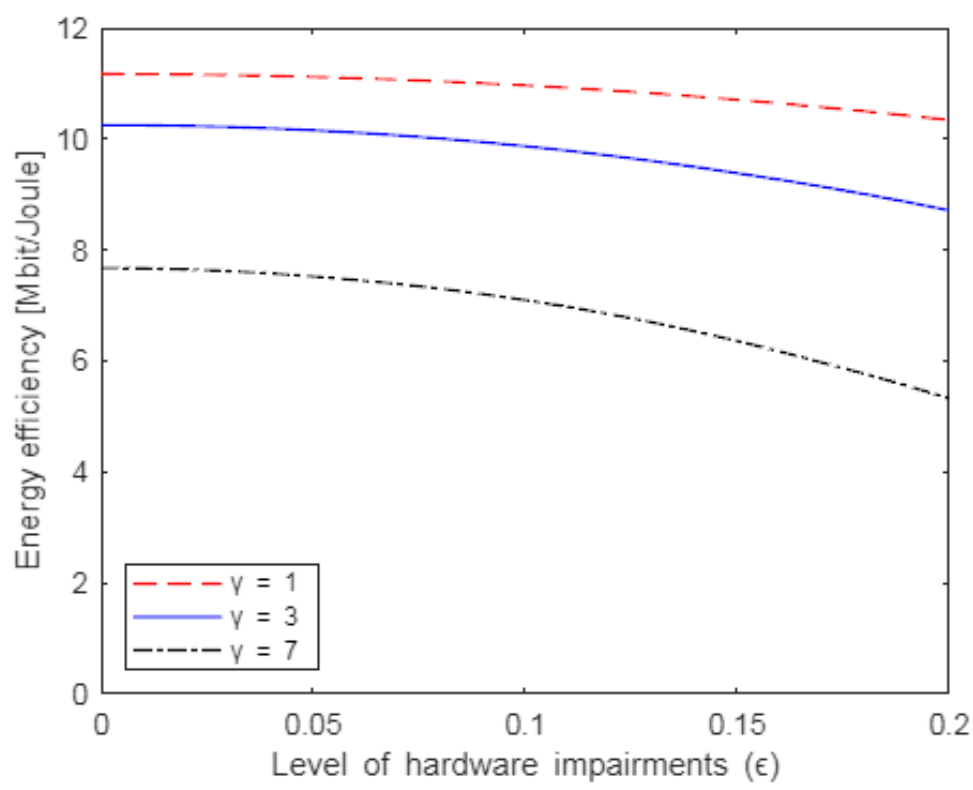

Fig. 6. Energy efficiency (in Mbit/J) as a function of the level of transceiver hardware impairments $(\varepsilon$ ), for different SINR constraints.

C. Effect of on user density on the energy efficiency of $5 \mathrm{G}$ small cells:

The aftermath on EE when evolving the number of user density in each irregular deployment cells has been simulated. Figure. 6 displays the EE has a function of the UE density for the typical SINR value comprises 3, while Figure. 7 displays the equivalent BS density when the number of BS antennas M, and the number of UEs $\mathrm{K}$ altered. The simulation is performed for massive multi-user MIMO transmission with $\mathrm{M}=91$ and 10 to analyze the outcome of evolving these specifications in contrasting with that results attained from the use of small cells composition on the $5 \mathrm{G}$ EE value. 


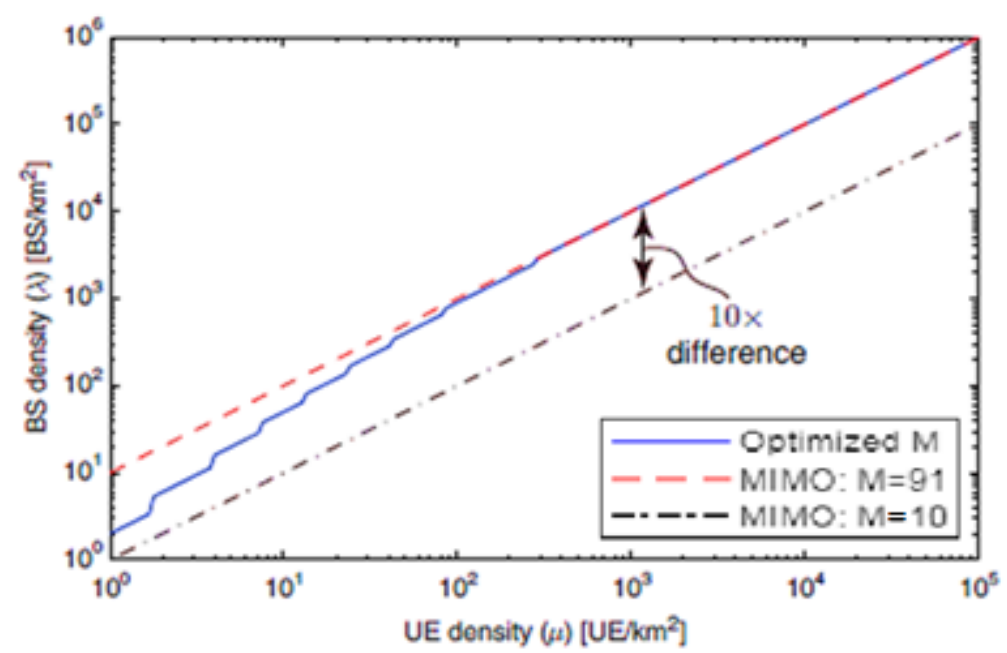

Fig. 7 Optimized BS density (in BS=km2) vs. the UE density (in UE=km2).

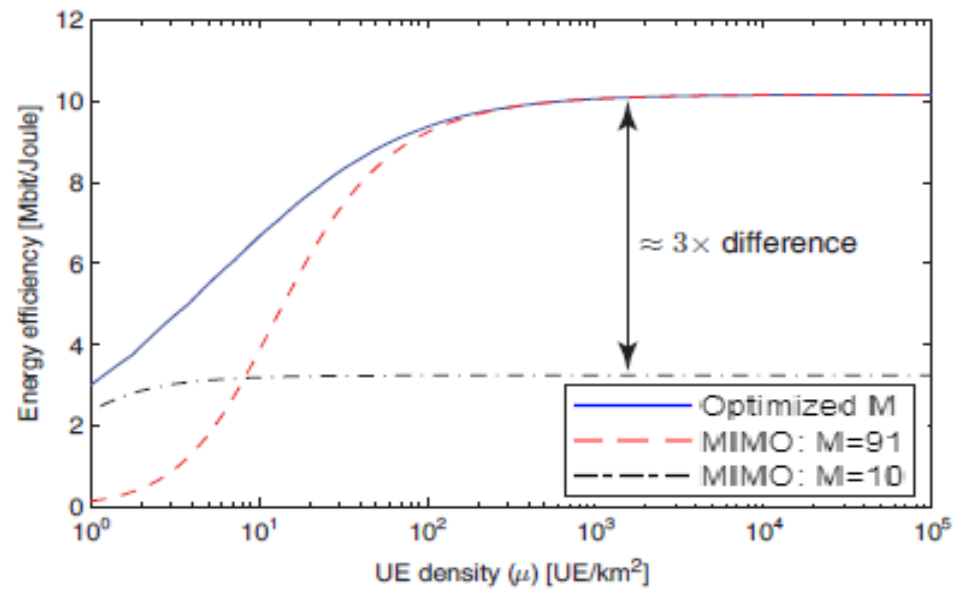

Fig. 8 Energy efficiency (in Mbit/J) vs. the UE density $\mu .(\alpha=4)$ Various conclusions are observed from the following outcomes demonstrated in Figure 3 and Figure 4.

- $\quad$ The first conclusion is that of the independency of the EE level on the UE density when the user density is comparatively large. Figure. 7 displays that the saturation values appear for user density more immense than 100 in both fixed and optimal massive MIMO situations. Depending on the results, it can simply scale c while exploiting the same number of UEs and BS antennas in each BS. Among all this, alterations can be obtained in the UE load by turning on and off the BSs.

- $\quad$ Also, figure 8. displays that the simulation of the steady massive MIMO framework accomplish the optimal EE in the saturation reign but ineffective when compared to low UE densities. In spite of its adequate performance at low UE densities, it saturates earlier and at a lower EE level compared to the maximal EE. Figure. 8 shows that the EE values increases to $11 \mathrm{Mbits} /$ joule when using 91 antennas, when using 50 small cells. At 10 antennas used, the EE declines by $72.7 \%$ and reaches 3 Mbits/joule. 
Vol. 10, Issue 7, July 2021

DOI 10.17148/IJARCCE.2021.10786

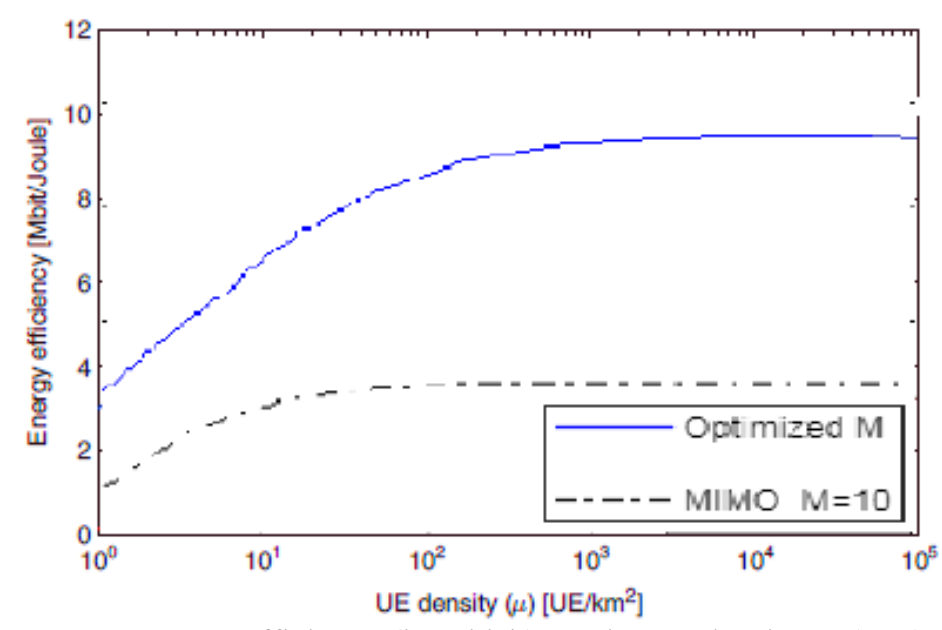

Fig. 9 Energy efficiency (in Mbit/J) vs. the UE density $\mu .(\alpha=3)$

As the energy efficiency rates extent, the same rates when employing small cells and massive MIMO. Furthermore, the choice of narrow coverage with simple frequency reuse, the small cells composition is desirable to employ but with restraints of the handover between cells. If the configuration requires to provide enormous number of users in a large coverage area, the massive MIMO composition is preferred but with a restraint of the complexity of the system and BS implementation. Moreover, Fig. 8 demonstrates that a single-user case demands for 10 times larger BS density in the saturation reign, which might extremely lower the implementation cost.

As in Fig. 9, it demonstrates the outcome of altering path loss coefficient on the EE in state of employing massive MIMO. The influence of diminishing the path loss coefficient illustrates that the EE declines as $\alpha$ declines. It decreases by $31.8 \%$ from the use of $\alpha=3$ and attains $7.5 \mathrm{Mbits} /$ joule. The figure also displays that altering the path loss coefficient does not influence the massive MIMO configuration at small number of antennas.

\section{VII.CONCLUSION}

In this paper, we have discussed the context of fifth-generation wireless technology. Additionally, Energy efficiency has now become a vital aspect in the framework of communication networks. With the introduction of the fifth generation of wireless networks, with millions of increased base stations and billions of connected devices, the persistence for energy-efficient system architecture and operation will be certainly more fascinating. Significantly, this paper reviews the diverse research work that gives an account of energy efficiency encompassing the papers published in this special concern configuration. Moreover, a theoretical context of the use of random waypoint mobility has been discussed. Studying the methodology for this simulation project has also been introduced with short outline about the simulation procedure. The simulation discussed in this paper presented that the EE is not affected by decreasing the cell size and is not the appropriate solution to influence a high EE. The most consequence on EE was observed when the BS density was increased, affecting the circuit power to lead the transmission power. It is cleared from the outcomes that EE decreases as the SINR values decreases and that is why there is a requirement to stipulate a target SINR to escape a situation of having an inefficient EE maximizing operation point. Furthermore, investigating energy-efficient massive MIMO systems in the Heterogeneous networks a leading challenge in the research field.

\section{REFERENCES}

[1]. J. G. Andrews, "Seven ways that HetNets are a cellular paradigm shift,” IEEE Communications Magazine, vol. 51, no. 3, pp. 136-144, March 2013.

[2]. W. Zhaocheng, C. Qian, L. Dai, J. Chen, C. Sun et al., "Location-based channel estimation and pilot assignment for massive MIMO systems," in IEEE Int. Conf. on Communication Workshop, London, UK, pp. 1264-1268, 2015.

[3]. D. Ha, K. Lee and J. Kang, "Energy efficiency analysis with circuit power consumption in massive MIMO systems," in IEEE 24th Annual Int. Sym. on Personal, Indoor, and Mobile Radio Communications, London, UK, pp. 938-942, 2013.

[4]. W. Liu, S. Han and C. Yang, "Is massive MIMO energy efficient?," arXiv preprint arXiv:1505.07187, 2015.

[5]. Peng, M., Zhang, K., Jiang, J., Wang, J., \& Wang, W. (2014). Energy-efficient resource assignment and power allocation in heterogeneous cloud radio access networks. IEEE Transactions on Vehicular Technology, 64(11), 5275-5287.

[6]. W. Zhaocheng, C. Qian, L. Dai, J. Chen, C. Sun et al., "Location-based channel estimation and pilot assignment for massive MIMO systems," in IEEE Int. Conf. on CommunicationWorkshop, London, UK, pp. 1264-1268, 2015.

[7]. Bai, Q., \& Nossek, J. A. (2015). Energy efficiency maximization for 5G multi-antenna receivers. Transactions on Emerging Telecommunications Technologies, 26(1), 3-14. 


\section{International Journal of Advanced Research in Computer and Communication Engineering}

Vol. 10, Issue 7, July 2021

\section{DOI 10.17148/IJARCCE.2021.10786}

[8]. Ge, X., Ye, J., Yang, Y., \& Li, Q. (2016). User mobility evaluation for 5G small cell networks based on individual mobility model. IEEE Journal on Selected Areas in Communications, 34(3), 528-541.

[9]. H. Q. Ngo, L. N. Tran, T. Q. Duong, M. Matthaiou and E. G. Larsson, "On the total energy efficiency of cell-free massive MIMO," IEEE Transactions on Green Communication and Networking, vol. 2, no. 1, pp. 25-39, 2017.

[10]. Prasad, K. S. V., Hossain, E., \& Bhargava, V. K. (2017). Energy efficiency in massive MIMO-based 5G networks: Opportunities and challenges. IEEE Wireless Communications, 24(3), 86-94.

[11]. J. Hoydis, S. T. Brink and M. Debbah, "Massive MIMO in the UL/DL of cellular networks: How many antennas do we need?," IEEE Journal Selection Areas Communication, vol. 31, no. 2, pp. 160- 171, 2013.

[12] N. P. Eugenio Ruggeri, Apostolos Tsakyridis,Christos Vagionas, Yigal Leiba, George Kalfas and A. Miliou, "Multi-user V-band uplink using amassive MIMO antenna and a fiber-wireless IFoF fronthaul for 5G mmWave small-cells," J. Light. Technol., vol. 1, no. 1, 2020.

[13] F. A. Ahmed Thair Al-Heety, Mohammad Tariqul Islam, Ahmed Hashim Rashid, Hasanain N. Abd Ali, Ali Mohammed Fadil, "Performance Evaluation of Wireless data traffic in Mm wave massive MIMO communication," Engineering, vol. 20, no. 3, 2020.

[14] C. F. D. Felipe A. P. de Figueiredo, E. R. de Lima, and and G. Fraidenraich, "Capacity Bounds for Dense Massive MIMO in a Line-of-Sight Propagation Environment," sensors, vol. 20, pp. 1-24, 2020.

[15] A. L. Swindlehurst, E. Ayanoglu, P. Heydari, and F. Capolino, "Millimeter-wave massive MIMO: The next wireless revolutison?," IEEE Commun. Mag., vol. 52, no. 9, pp. 56-62, 2014.

[16] N.-C. Chuang, H.-S. Lin, and Y. Lin, "Compact cavity-backed dual-polarized aperture antennas for millimeter wave MIMO applications," in 2017 IEEE MTT-S International Conference on Microwaves for Intelligent Mobility (ICMIM), 2017, pp. 131-134.

[17] E. Kurniawan and A. Goldsmith, "Optimizing cellular network architectures to minimize energy consumption," in Proc. of IEEE International Conference on Communications (ICC), 2012, pp. 4771-4775.

[18] W. Liu, S. Han, C. Yang, and C. Sun, "Massive MIMO or small cell network: Who is more energy efficient?," in Proc. of IEEE Wireless Communications and Networking Conference Workshops (WCNCW), 2013, pp. 24-29. 